\title{
Myricitrin Attenuates Hypoxic-ischemic Brain Injury in Neonatal Rats by Mitigating Oxidative Stress and Nrf2/HO-1/ARE Signaling Pathway
}

Ting Yan

Third hospital of Jinan https://orcid.org/0000-0002-0724-6965

Man Lu ( $\nabla$ manlu673@yahoo.com )

Taian City Central Hospital

\section{Original article}

Keywords: Antioxidant, Hypoxic-ischemic (HI), Myricitrin, Neuroinflammation

Posted Date: August 25th, 2020

DOl: https://doi.org/10.21203/rs.3.rs-58802/v1

License: @ (1) This work is licensed under a Creative Commons Attribution 4.0 International License.

Read Full License 
1 Myricitrin attenuates hypoxic-ischemic brain injury in neonatal rats by mitigating

2 oxidative stress and Nrf2/HO-1/ARE signaling pathway

3 Ting $\operatorname{Yan}^{1}, \operatorname{Man} \mathrm{Lu}^{2 *}$

$4 \quad{ }^{1}$ Department of Neurology, The Third Hospital of Jinan, Jinan, 250132, Shandong, China.

$5 \quad$ Email: mepshp@ 163.com

$6 \quad{ }^{2}$ Department of Pediatric, Taian City Central Hospital, Taian, 271000, Shandong, China

7 Email: manlu673@yahoo.com

8

$9 \quad *$ Corresponding author:

10 Man Lu, Department of Pediatric, Taian City Central Hospital, No. 29 Longtan Road,

11 Taian271000, Shandong, China

12 Tel/Fax: 0086-05388223227

13 Email: manlu673@yahoo.com

14

15

Running title: Neuroprotective effect of myricitrin

16

17

18

19

20 
23

24

\section{Abstract}

The present study has been explored the neuroprotective efficacy of myricitrin and unveil the mechanism of action via the regulation of $\mathrm{Nrf} 2 / \mathrm{HO}-1 / \mathrm{ARE}-$ dependent signaling cascade in hypoxic-ischemic (HI) induced brain injury of neonatal rats. Upon HI operation neonatal rats received 20 and 40mg/kg bw of myricitrin as intraperitoneally up to one week twice in a day. Myricitrin administration augmented HI induced increase of infarct volume and oedema of the brain, as well as improve neurobehavioral impairments. Further, we measured the level the antioxidant enzymes and oxidative stress marker level of ROS, NO and product of lipid peroxidation. These data support the well-established radical scavenging and antioxidant potential of myricitrin corresponds to a significant decline in most vulnerable radical formation with an obvious increase in antioxidant enzyme content. Interestingly, myricitrin source a significant reduction in NF- $\mathrm{BB}$ p65 and inflammatory marker gene expression, while reducing levels of the nuclear fraction of Nrf2- antioxidant response element (ARE) and cytosolic fraction of HO-1 enzyme activation. The results substantiate the efficiency of myricitrin an activation of Nrf2/ARE-dependent HO-1 pathway via improve defense mechanism and mitigate oxidative stress associated neuroprotective effect.

Key words: Antioxidant, Hypoxic-ischemic (HI), Myricitrin, Neuroinflammation, 


\section{Introduction}

Neonates are succumbed to Hypoxic Ischemic (HI) encephalopathy which causes neuronal damage during intrapartum asphyxia and it accounts for about $25 \%$ of infant mortality globally (Varghese et al. 2016). Perinatal (HI) encephalopathy remains a common cause of socio-economic burden and a major cause of disability in infants resulting in motor and learning impairment, epilepsy, seizures and eventually cause death (Hossain. 2008). Perinatal HI has been well-defined to be associated with an array of events contribute to neuronal damage, largely generation of free radicals and loss of ATP, excitotoxicity and failure of calcium export/import are being the other factors (Qin et al. 2019). This abnormal increase in the superoxide dismutase (SOD) and Glutathione peroxidase (GPx) level has led the cells make it prone to the formation of free radicals and excess lipid peroxidation. Moreover, increased iron $\left(\mathrm{Fe}^{2+}\right)$ levels in neonatal brain favors the ROS generation via Fenton's reaction, catalyzing the generation of hydroxyl radical $(\mathrm{OH})$ ion being the highly oxidative agent which cause severe brain damage (Aridas et al. 2014; Ten and Starkov. 2012), Heme oxygenase-1 (HO-1), is one of the antioxidant enzymes attenuates oxidative stress-mediated inflammation and alleviates neuronal loss with well-known cytoprotective effects (Chao et al.2014; Piras et al. 2017). Studies concluded that patients diagnosed with Parkinson Disease (PD) has identified elevated HO-1 in serum relative to chronic oxidative stress (Mateo et al. 2010).

In eukaryotic organisms, cellular redox balance is regulated by transcription factor nuclear factor erythroid 2-related factor 2 (Nrf2), which is encoded by $N f e 2 l 2$ gene. Nrf2 binding with antioxidant response element (ARE) present in the HO-1 promoter region (Park et al. 2013). The abnormality in Nrf2 mediated defense at ageing is thought to be one risk factor to 
cause PD and found to be accompanied with diminished Nrf2/ARE-dependent cytoprotective enzyme activity levels (Kumar et al. 2012; Zhang et al. 2013; Francisqueti-Ferron et al. 2019; Jiang et al. 2017). Despite, the number of therapeutic options in clinics, none of them has exerted a complete cure for HI. Therefore, it is suggested that strategies aimed at preventing and or reducing neuronal damage or neuronal cell death will be warranted to reduce the disabilities associated with HI encephalopathy.

Flavonoids are large groups of a polyphenolic moiety, Myricitrin is one among the polyphenol called hydroxy flavonoid in terms of IUPAC is $3^{\prime}, 4^{\prime}, 5^{\prime}, 5$, 7-five hydroxy flavone-3-O- $\alpha$-L-rhamnoside (Figure 1) whose natural presence is reported in various parts of bayberry include fruits, leaves, bark and stem as well as in the root bark of Myrica species of plants (Liao et al. 2006; Liu et al. 2010; Aloud et al. 2017). Myricitrin has reported for its various beneficial pharmacological activities such as anticancer (Lee et al. 2007; Kale et al. 2008), antimicrobial, antiviral (Cushnie et al. 2005) and antinociceptive(Meotti et al. 2006), In lieu with literature, myricitrin shows highly defensive on free radical scavenging and prooxidant defense than other counterparts such as quercetin and rhamnosides (Wu et al. 2008).

However, studies with respect to neuroprotection under normal and hypoxic or pathological responses were Nrf2/HO-1/ARE axis has just been the intense research theme recently. Thus, the role of myricitrin on impact the signaling system identified with inflammable occasions in hypoxia incited brain injury in neonatal rodents have not been researched sufficiently. In this experiment, hypoxia induced-ischemic brain injury is established in neonatal rat pups to evaluate the anti-inflammatory potential of myricitrin. Further, to explore the molecular mechanisms, we emphasized the Nrf2/HO-1/ARE axis.

\section{Materials and Methods}


94 Seven days-old female Sprague-Dawley rats were free of pathogens procured from Animal

95 Experimental Center of Ningxia Medical University, Yinchuan, China (Permit number: 96 SCXK Ningxia 2015-0001). Experiments involving neonatal rats were approved by the Taian 97 City Central Hospital Institutional Animal Ethical Committee. Animals were treated in accordance with Guide for the Care and Use of Laboratory Animals (8th edition, National Academies Press). All rats were housed in individual cages under constant regulated day/light cycles $(6: 00 \mathrm{AM}$ to $6: 00 \mathrm{PM})$, under controlled temperature $\left(21^{\circ} \mathrm{C}\right)$. were provided free access to water and food ad libitum. All the efforts were made to minimize the animals suffering and usage. Pups from day 7 were chosen for experimental purpose as they are comparable to 32 to 34th-week neonates of humans (Vannucci and Hagberg 2004). Neonatal rats were randomly picked and divided into four different groups for experimental purposes.

\section{Experimental Groups}

Animals were acclimated for about 1 week before experiments. To investigate the neuroprotective efficiency of myricitrin during HI neuronal damage in perinatal infant rats, the animals were arbitrarily divided into four different groups.

Group I Sham $(\mathrm{n}=12)$

Group II $\quad$ HI induced + Saline administered $(n=12)$

Group III HI induced + Myricitrin administered (Myricitrin was dissolved in saline contains 2\% DMSO and administered intraperitoneally (ip) at a dose of $20 \mathrm{mg} / \mathrm{kg}$ body weight until the end of the experiment $(n=12)$. 
Group IV $\quad \mathrm{HI}$ induced + Myricitrin a dose of $40 \mathrm{mg} / \mathrm{kg}$ body weight administered as ip to pups until the end of the experiment $(\mathrm{n}=12)$.

Hypoxic Ischemia (HI) was induced as follows: Briefly, 7-days-old (P7) offspring pups were administered 2-3\% isoflurane to anaesthetize via the inhalation. Carotid artery 119 (CCA) at right ventricle was surgically exposed, was double ligated using 5.0 silk surgical sutures and then cut was open in between two ligation sites. Animals were recuperated upon surgery for 1.5 hours and put into the hypoxic incubator (with $92 \%$ nitrogen balanced with $8 \%$ oxygen for 1.5 hours at $37^{\circ} \mathrm{C}$ ). $8 \%$ oxygen-balance nitrogen is widely used to induce hypoxic ischemia via inhalation to rat pups (Wang et al. 2017). Finally, animals were allowed to recover upon the hypoxic treatment. For sham treatment groups, only CCA was exposed but not undergone ligation and the hypoxic treatment.

Myricitrin was obtained from Selleckchem-S232701 (Shanghai, China) and dissolved in sterile physiological saline contains $2 \%$ DMSO to obtain desire concentration and were administered to rat pups. Myricitrin was administered for every $12 \mathrm{~h}$ for 7 consecutive days after induction of HI injury. Sham and HI pups were treated with saline with $2 \%$ DMSO as vehicle control. At the end of 7 days of experimental regime neonatal rats were sacrificed under pentobarbital sodium $50 \mathrm{mg} / \mathrm{kg}$ ip and $4 \%$ paraformaldehyde was used for brain tissue fixation for histological analysis (six rats from each group). phosphate buffer for lysis from Sigma-Aldrich; MO, USA used for homogenate preparation for biochemical as well as molecular biology analysis ( $\mathrm{n}=6$ each group).

\section{Measurement of brain infarct volume}

Brain infarct volume was measured using 2,3,5-triphenyltetrazoliumchloride (TTC) stain after 24 hours upon 7 days of $\mathrm{HI}$ as followed previously described methods with some modifications (Jin et al. 2015). In detail, 4\% isoflurane was used to anaesthetize the rat pups 
and sacrificed then the brain was removed and kept frozen before staining. $2 \mathrm{~mm}$ thickness of coronal sections of brain tissue obtained by microtome of Leica Microsystems. (Wetzlar at Germany) and was incubated with $2 \%$ TTC at $37^{\circ} \mathrm{C}$ for $20 \mathrm{~min}$. Then $4 \%$ paraformaldehyde is used for fixation for overnight at $4{ }^{\circ} \mathrm{C}$ were further washed with PBS. The brain damage (contralateral hemisphere damage percentage) was quantified using the Image-Pro plus software USA version of 7.0.

\section{Measurement of brain cerebral oedema}

Wet (24 h after the $\mathrm{HI}$ and treatment) and dry weight of the brain (after drying at $105{ }^{\circ} \mathrm{C}$ for $24 \mathrm{~h}$ ) were measured. The brain oedema was calculated as follows: (wet weight - dry weight)/wet weight $\times 100 \%$.

\section{Coordination deficits and motor activity measurement}

RotaRod experiment was conducted after 14 days of HI to measure the coordination deficit and motor activity as followed previously mentioned (Pazos et al. 2012). Two days of training; First day $4 \mathrm{rpm}$ (revolutions per minute) for $1 \mathrm{~min}$ and second day 4 to $40 \mathrm{rpm}$ for 10 min maximum of the time. Upon completion of training two times of trails, $1 \mathrm{~h}$ rest between each were conducted. The latency to fall in all rat pups was recorded in seconds.

\section{Measurement of ROS and lipid peroxidation and antioxidants}

DCFH-DA (2'-7'-dichlorofluorescein-diacetate) assay was performed to quantify the level of ROS (Siqueira et al. 2005), where the oxidation product of DCFH to the fluorescent $2^{\prime}, 7^{\prime}$-dichlorofluorescein (DCF) the formation is used to quantify ROS. The development of DCF was measured by spectrofluorimetry (Excitation wavelength: $504 \mathrm{~nm}$; Emission wavelength: $529 \mathrm{~nm}$ ). Total nitric oxide (NO) production was measured by 
spectrophotometrically were the accumulation of nitrite and nitrate in tissue homogenates is quantified by Griess reagent (Total Nitric Oxide Assay Kit of Beyotime, Jiangsu, China). For protein estimation, Pierce protein assay kit obtained from Thermo Fisher Scientific Inc., MA, USA used to measure the protein concentration of brain tissue homogenate. The tissue levels of malondialdehyde (MDA), a product of lipid peroxidation: MDA-A003 superoxide dismutase: SOD-A001, catalase: CAT-A007, GSH-glutathione peroxidase: GPX-A017 was used to measure the antioxidant levels based on manufacture instruction using a commercial micro test kits obtained from Nanjing Jiancheng Bioengineering Institute at Nanjing, China.

\section{Analysis of Gene Expression}

RNA isolator reagent from Vazyme Biotech Co., Ltd., was used for total RNA isolation adhere with the instructions from manufacture. HiScript ${ }^{\circledR}$ II 1st Strand cDNA Synthesis kit used to perform reverse transcription reaction. AceQ qPCR SYBR Green Master Mix kit was used for Real-time PCR experiment by using the Bio-Rad CFX System and following the method described previously (Guruvaiah et al. 2018). The primers used for mice gene expression listed in Table 1 obtained from Vazyme Biotech Co., Ltd., Nanjing, China).

\section{ELISA quantification of inflammatory marker proteins}

Quantification of various proinflammatory molecules, expression was determined in cerebral tissue homogenate by using commercially available ELISA micro-test kits obtained from Biovision Inc, CA, USA.

\section{Nrf2 and HO-1 expression analysis by blotting}

Analysis of nuclear fraction of Nrf2 and cytosolic fraction of $\mathrm{HO}-1$ in brain cerebral cortex was measured using respective nuclear and cytosolic kits (Biovision Inc., CA, USA) followed manufactures instructions. The concentration of extracted protein was estimated by using a BCA protein assay kit purchased from Beyotime, China. 10\% SDS-PAGE gel used for 
separation of the protein $(40 \mu \mathrm{g} / \mathrm{lane})$, and the separated protein electroblotted onto poly-

187 vinylidene difluoride (PVDF) membranes obtained from Millipore, Bedford, MA). TBST (Tris-buffered saline with $1 \%$ Tween 20) solution contains $5 \%$ of skimmed milk was used as a blocking solution to block the membrane from false signals. This membrane has subsequently incubated $\left(4^{\circ} \mathrm{C}\right.$ overnight $)$ with primary antibody of rabbit polyclonal anti-Nrf2 (G921a Promega Corp., Madison, WI, USA) and -HO-1 of 1:500 and 1:800 dilution respectively (CD143 Stressgen Biotechnologies, Victoria, BC, Canada) and anti-rat $\beta$-actin (polyclonal-rabbit) of 1:500 dilution was obtained from Beijing Zhongshan Goldenbridge Biotechnology, China. Subsequently, membranes were incubated with horseradish peroxidase (HRP) conjugated secondary antibody (1:10,000-Abcam, Cambridge, UK) diluted with TBS at room temperature for $60 \mathrm{~min}$. The band absorbance was quantified using DAB and band intensity was measured by using Image J software developed by NIH, Bethesda, MA.

\section{Hematoxylin and Eosin staining}

4\% paraformaldehyde in phosphate-buffered saline (PBS) is used to fix the brain tissue excised from brain isolated upon completion of the experiment. Overnight incubation in paraformaldehyde at $4^{\circ} \mathrm{C}$ and embedded in paraffin wax. The 5- $\mu \mathrm{m}$ thickness of coronal sections of brain tissue was cut using a microtome. Finally, the microtome sections were stained with hematoxylin and eosin (H\&E)

\section{Statistical Significance}

GraphPad Prism 8.0 was used for statistical analysis and results were presented as mean \pm 
significance of differences between experimental groups for multiple data comparison. Neurological function statistical analysis we use non-parametric analysis. $\mathrm{P}<0.05$ value was considered to be significant.

\section{Results}

\section{Myricitrin administration reduces HI induced cerebral infarct area in brain}

Area of cerebral infarct in rat brain was measured by using TTC stain (Figure 2). The CI area appears white patches in the HI group, were increased $(38.15 \pm 2.11 \%)$ significantly $(p<0.05)(33.85 \pm 3.15 \%)$ than those of the sham control group. However, the myricitrin administered groups of rat brain showed the CI area of $25.21 \pm 1.65 \%$ and $16.30 \pm 2.13 \%$, of 20 and $40 \mathrm{mg} / \mathrm{kg}$ bw respectively. This observation indicating that myricitrin could noticeably control the spread of HI-induced CI area associated with brain damage.

\section{Myricitrin reduces cerebral oedema in $\mathrm{HI}$ induced rat brain}

$\mathrm{CO}$ of $\mathrm{HI}$ induced rat brain tissue was drastically $(p<0.05)$ increased as 81.23 vs. 0 in the sham group (units in \%). Myricitrin administration (20 and $40 \mathrm{mg} / \mathrm{kg}$ ) control the $\mathrm{CO}$ and keeps the $(p<0.05)$ the level of $\mathrm{CO}$ in 68.16 and $61.42 \%$, respectively (Fig. 2), in that way exert its neuroprotective action reducing the occurrence of water in brain tissue were those compared with the HI group.

\section{Myricitrin improves coordination and motor activity in $\mathrm{HI}$ induced rats}

RotaRod test was carried out to identify the coordination and motor activity of rats. HI, induced rats show poor activity with a very less time spent on the rod compared those of animals in Sham groups (Fig. 2). Myricitrin administration to rat pups has improved the 
latency to fall those compared with sham (Fig 2). When a higher dose of myricitrin administration has an increase in time spent on the rod.

\section{Myricitrin improves antioxidant status in $\mathrm{HI}$ induced rat brain}

Product of lipid peroxidation (MDA), nitric oxide (NO) and various antioxidant enzymes were analysed to validate the effect of myricitrin on neuroprotection of HI-induced rat pups brain against ROS induced oxidative stress (Fig. 3). The activity level of GSH, GPx, CAT and SOD were significantly declined in HI induced brain those compared sham group, Thus the lipid peroxidation product of MDA was considerably increased $(p<0.05)$. Myricitrin treatment $(20$ and $40 \mathrm{mg} ; p<0.05)$ significantly restore the activities of antioxidant enzymes as well as a beneficial decline in formation of MDA and nitric oxide in brain tissues.

\section{Myricitrin administration down-regulate the inflammatory gene expression in $\mathrm{HI}$} induced rat brain

Effect of myricitrin treatment on inflammatory marker genes in HI induced brain tissue has determined my real-time PCR. The mRNA expression pattern of several proinflammatory cytokines also measured to confirm the signalling regulation of inflammation. Fig. 2, shows $\mathrm{HI}$ insult resulted in mRNA overexpression of TNF $\alpha$, IL-1 $\beta$, IL-6, HO-1 and p65 NF- $\kappa$ B. Intraperitoneal administration of myricitrin to $\mathrm{HI}$ induced rat pups shows controlled and downregulation of proinflammatory cytokines and HO-1 (Fig. 4). The higher dose of myricitrin shows a more significant protective effect on the expression of inflammatory and oxidative stress markers the results support the effect of myricitrin on reducing inflammation after HI operation.

\section{Myricitrin inhibits inflammation induced by $\mathrm{HI}$ in the brains of rat pups}


Downregulation of inflammatory marker genes mRNA expression was demonstrated by realtime PCR, further confirmation the translational product concentration was quantified by ELISA. The effect of myricitrin on marker protein after HI induction was assessed by evaluating the levels of oxidative stress mediated ARE and inflammatory associated proinflammatory cytokines like IL-6, IL-1 $\beta$ and TNF- $\alpha$ as well as NF- $\kappa B$ free p65 subunit (Table 2). HI induced rat brain tissue shows statistically pronounced level of $(p<0.05)$ increase in inflammatory marker protein molecules (HO-1, IL-6, IL-1 $\beta$, TNF- $\alpha$ and NF- $\kappa$ B free p65) than those of sham control group. Treatment with Myricitrin treatment significantly restore the $(p<0.05)$ the HI-induced abnormal increase in the level of these inflammatory markers. Thus, it could be noted that the anti-inflammatory effect of the comparably better in higher dose myricitrin treated rat brain tissue.

\section{Myricitrin augments protein expression of nuclear Nrf2 and cytosolic HO-1 in the} cerebral cortex of rats

Blotting analyses were performed to confirm the effects of myricitrin on the protein expression pattern of HO-1 of cytosolic and Nrf2 of nuclear (Fig 5). The protein expression of Nrf2 and HO-1 in the cerebral cortex of HI, subjected rat brain was unusually upregulated $(p<0.05)$ where on equivalence to sham control group. Of note, myricitrin treatment $(20$ or 40) reverse $(p<0.05)$ the upregulated expression of HO-1 and Nrf2 after HI insult.

\section{Hypoxic-ischemia induced brain damage normalized by myricitrin}

The neuroprotective efficiency of myricitrin on HI induced brain damage was observed in HE stained brain tissue. Sham group tissue sections showed normal intact round shaped cell bodies, whereas HI group tissue sections has observed atrophic cell bodies in the intense staining sections. Thus, HI induced myricitrin treated neonatal brain tissue shows reduced 
cell atrophy and resembles near normal morphology (Fig 6). In brief, identify the dose dependent effect of myricitrin, brain tissue of high dose of myricitrin received neonates shows normal cellular morphology indicates myricitrin produce enhanced protective effect with respect to doses tested.

\section{Discussion}

Myricitrin is a flavone class of bioactive compound occurring naturally in various herbs and studies explored its efficiency in various pharmacological activity both in vivo and invitro. Studies confirmed that myricitrin could activate Nrf2-mediated HO-1 and NQO-1, further mediate anti-oxidant signaling and attenuates HG-induced H9c2 cell apoptosis via activation of Akt signaling. It should be better to explain that a number of studies clearly demonstrated that Nrf2 nuclear translocation can be strongly persuaded by phosphatidylinositol 3kinase/protein kinase B (PI3K/Akt) signaling cascade activation. Thus, activation of the PI3K/Akt pathway can subsequently modulate the Nrf2 mediated HO-1 expression has been considered a possible approach to hinder HI induced neuronal damage (Zhang et al. 2016). Interestingly, it was reported that myricitrin possess remarkable anti-inflammatory and antifibrotic properties (Domitrović et al. 2015). Nonetheless, the effects of myricitrin on HI induced brain damage in the newborn and its protective mechanism has not yet been studied. This is the maiden report on myricitrin about attenuate the HI-induced brain injury and further demonstrate the role of Nrf2/HO-1/ARE signalling pathway. Scientific studies clearly hypothesized that ROS mediated oxidative stress and consequent inflammatory responses upon HI induction being the key mediators of pathological change associated with HIE (Lu et al 2012; Arteaga et al. 2014).

In general, in vivo studies of brain cerebral ischemic condition, the degree of damage in neuronal part is assessed by brain infarct volume measurement (Fukui et al. 2002). In our 
experimental measurement of brain infarct volume and oedema, the pups subjected to HI had developed a more infarct area and a higher rate of water accumulation become the signs of oedema and inflammatory neuronal damage and response. Coordination and motor activity observation study exhibit poor time spent on RotaRod test indicates HI induced rats has not better in coordination and activity. In contrast, pups received myricitrin has improved in coordination and the latency to fall those compared with HI and near equal to sham. Our results regarding infarct volume area have been agreed with the findings of Karalis et al. (2011) of noted that resveratrol treatment to HI induced rat pups has significantly decreased the infarct area of the brain through preventing the demyelination (Yousuf et al. 2009). This ameliorative effect would highly correlate with the observed pathological changes made by myricitrin.

The neonatal brain is more susceptible to ROS mediated oxidative stress than those of adult counterpart and it has high polyunsaturated fatty acids content and utilizes more oxygen favours (PUFA) subsequent antioxidants deficiency. This ideal property of the neonatal brain made itself more susceptible to cause a pathological increase of free radical generation, subsequently, it causes neuronal damage in brain tissue of neonatal rat (Yousuf et al. 2009; Habtemariam et al. 2019).

In order to identify the role of myricitrin in antioxidant profile and oxidant defence, we measured the level of cellular antioxidant enzymes. Myricitrin administration significantly improves the status of antioxidant and reduce the rate of lipid peroxidation and formation of NO. This data strongly supports the reported antioxidant role of myricitrin in various mechanistic studies. The experimental studies on neuroinflammation through the direct supply of ROS or 6-OHDA (6-hydroxydopamine) and MPTP (1-methyl-4-phenyl-1,2,3,6tetrahydropyridine-MPTP) to establish Parkinson's disease (PD) or through the A $\beta$ model of Alzheimer disease (AD), were pinocembrin is flavonoid class of compound exhibits 
neuroprotection via the oxidative stress improvement. This evidence could support to correlate the myricitrin that mediates antioxidant defence in $\mathrm{HI}$ induced brain damage. In these studies, the mechanism of neuroprotection has been achieved via the antioxidant defence improvement can subsequently induce the HO-1 enzyme activity thereby activation of Nrf2. Meanwhile, irrespective to neuroprotection pinocembrin could mitigate the oxidative stress through Nrf2-HO-1 signaling in carbon tetrachloride- $\left(\mathrm{CCl}_{4}-\right)$ induced liver fibrosis and nephrotoxicity (Karalis et al. 2011).

Most of the studies about flavonoid group of compounds in neuroprotection could be regulated by Nrf2 and its transcriptional activator HO-1 being antioxidant response elements (ARE) by using the SH-SY5Y cellular model (Yousuf et al. 2009). Moreover, studies mention that flavonoids could also inhibit the expression of $\mathrm{NF}-\kappa \mathrm{B}$, inducible nitric oxide synthase (iNOS), cyclooxygenase-2 (COX-2) and proinflammatory cytokines include TNF- $\alpha$, IL-1 $\beta$, IL-6. We measured the mRNA expression pattern of inflammatory molecules that shows myricitrin can control the overexpression of these genes and the data of inflammatory cytokines quantification also well connected with the mRNA expression pattern.

Neurotoxicity induced by various neurotoxic agents such as $\mathrm{H}_{2} \mathrm{O}_{2}$, paraquat, methylglyoxal and 6-hydroxydopamine (6-OHDA) could ameliorate efficiently by well-known flavonoid compound pinocembrin. The experimental neuroprotection via the constructive role of Nrf2HO-1 axis was evidenced by the Nrf2 silencing study or HO-1 inhibition by ZnPP IX of $0.5 \mu \mathrm{M}$. In addition, it has been shown that flavonoids exert anti-inflammatory effect against lipopolysaccharide- (LPS) and $\mathrm{H}_{2} \mathrm{O}_{2}$ induced inflammatory damage in $\mathrm{BV}-2$ microglial cells and SH-SY5Y cells respectively through the Nrf2-HO-1 axis (Wang et al. 2016). Identify the role of myricitrin on Nrf2-HO-1-ARE axis in $\mathrm{HI}$ induced neuronal damage that we measure the protein expression of the nuclear fraction of Nrf2 and cytosolic fraction of $\mathrm{HO}-1$ in brain cerebral cortex. Myricitrin administration significantly suppress the Nrf2 and HO-1 
expression that demonstrates the translocation of Nrf2 inhibited by myricitrin thereby further overexpression of antioxidant response element-HO-1 also inhibited.

353 The present study was focus on neuroprotective effects of myricitrin on rat pups subjected to 354 HI insult, study has certain limitations and yet to identify the interlink between Nrf2/HO-1 associated other signaling cascade mechanism. The present study focused on ROS induced oxidative stress, antioxidant status and inflammatory signalling response. We did not asses addressed the other contributors to HIE such as apoptosis, mitochondrial dysfunction as well as its relationship with bioenergetics, future studies has planned to substantiate the mechanism. Accordingly, it could be suggesting that myricitrin be used for inflammatory neurological abnormalities treatment and the Nrf2/HO-1/ARE being the mechanistic pathway via that attenuate hypoxia-induced ischemic brain damage in neonatal rats. In-depth research on human clinical studies warranted for a more interesting conclusion.

\section{Acknowledgements}

The authors appreciate the help from the central laboratory of Taian City Central Hospital, Taian. P.R.China.

\section{Declaration of conflicting interests}

367 The author(s) declared no potential conflicts of interest with respect to the research, 368 authorship, and/or publication of this article.

\section{$369 \quad$ Funding}

370 No external funding received for this study.

\section{Authors contribution statement}


372 TY and ML conducted experiments and collected data. TY carried out data interpretation,

373 review of literature and manuscript drafting. ML prepare the manuscript final version and

374 both the authors read and approved the manuscript.

\section{Ethical approval statement}

376 Animal experiments were approved by the ethical committee of Taian City Central Hospital,

377 (No: 2019-20/ML 2231) Taian, 271000, Shandong, China.

378 Consent for publication

379 NIL

380 Availability of data and material

381 The Corresponding author could provide the all experimental data upon valid request.

\section{References}

Aloud AA, Veeramani C, Govindasamy C, Alsaif MA, El Newehy AS, Al-Numair KS

(2017) Galangin, a dietary flavonoid, improves antioxidant status and reduces hyperglycemia-mediated oxidative stress in streptozotocin-induced diabetic rats. Redox Rep 22(6):290-300. doi: 10.1080/13510002.2016.1273437. PMID: 28030991.

Aridas JD, Yawno T, Sutherland AE, Nitsos I, Ditchfield M, Wong FY, Fahey MC, hypoxic ischemic encephalopathy: closing the gap between experimental and clinical research. Exp Neurol 261:281-90. doi: 10.1016/j.expneurol.2014.07.009. PMID: 25079368. 

Antioxidants in Neonatal Hypoxic-Ischemic Brain Injury: New Therapeutic Approaches. Int J Mol Sci 18(2):265. doi: 10.3390/ijms18020265. PMID: 28134843.

Chao XJ, Chen ZW, Liu AM, He XX, Wang SG, Wang YT, Liu PQ, Ramassamy C, Mak SH, Cui W, Kong AN, Yu ZL, Han YF, Pi RB (2014) Effect of tacrine-3-caffeic acid, a novel multifunctional anti-Alzheimer's dimer, against oxidative-stress-induced cell death in HT22 hippocampal neurons: involvement of Nrf2/HO-1 pathway. CNS Neurosci Ther 20(9):840-50. doi: 10.1111/cns.12286. PMID: 24922524.

Cushnie TP, Lamb AJ (2005) Antimicrobial activity of flavonoids. Int J Antimicrob Agents 26(5):343-56. doi: 10.1016/j.ijantimicag.2005.09.002. PMID: 16323269. Myricitrin exhibits antioxidant, anti-inflammatory and antifibrotic activity in carbon tetrachloride-intoxicated mice. Chem Biol Interact 230:21-9. doi: 10.1016/j.cbi.2015.01.030. PMID: 25656916. Moreto F, Ferreira ALA, Minatel IO, Correa CR (2019) Basic Concepts on the Role of impairment of rats caused by oxidative stress and aging, and its prevention by vitamin E. Ann N Y Acad Sci 959:275-84. doi: 10.1111/j.1749-6632.2002.tb02099.x. PMID: 11976202. 

Abundant Sanglan Tea on Long-Term High-Fat-Diet-Induced Obesity Complications in C57BL/6 Mice. Nutrients 10(9):1276. doi: 10.3390/nu10091276. PMID: 30201876. Hossain MA. 2008. Hypoxic-ischemic injury in neonatal brain: involvement of a novel neuronal molecule in neuronal cell death and potential target for neuroprotection. Int $\mathbf{J}$ Dev Neurosci, 26(1):93-101. doi: 10.1016/j.ijdevneu.2007.08.013. PMID: 17936538. Jiang S, Deng C, Lv J, Fan C, Hu W, Di S, Yan X, Ma Z, Liang Z, Yang Y (2017) Nrf2 Weaves an Elaborate Network of Neuroprotection Against Stroke. Mol Neurobiol 54(2):1440-1455. doi: 10.1007/s12035-016-9707-7. PMID: 26846360.

Jin Z, Liang J, Wang J, Kolattukudy PE (2015) MCP-induced protein 1 mediates the minocycline-induced neuroprotection against cerebral ischemia/reperfusion injury in vitro and in vivo. J Neuroinflammation 12:39. doi: 10.1186/s12974-015-0264-1. PMID: 25888869.

Kale A, Gawande S, Kotwal S (2008) Cancer phytotherapeutics: role for flavonoids at the cellular level. Phytother Res 22(5):567-77. doi: 10.1002/ptr.2283. PMID: 18398903.

Karalis F, Soubasi V, Georgiou T, Nakas CT, Simeonidou C, Guiba-Tziampiri O, Spandou E (2011) Resveratrol ameliorates hypoxia/ischemia-induced behavioral deficits and brain injury in the neonatal rat brain. Brain Res 1425:98-110. doi: 10.1016/j.brainres.2011.09.044. PMID: 22018692.

Kumar H, Koppula S, Kim IS, More SV, Kim BW, Choi DK (2012) Nuclear factor erythroid 2-related factor 2 signaling in Parkinson disease: a promising multi therapeutic target against oxidative stress, neuroinflammation and cell death. CNS Neurol Disord Drug Targets 11(8):1015-29. doi: 10.2174/1871527311211080012. PMID: 23244425. 
Lee ER, Kang GH, Cho SG (2007) Effect of flavonoids on human health: old subjects but new challenges. Recent Pat Biotechnol 1(2):139-50. doi: 10.2174/187220807780809445. PMID: 19075837.

Liao HW, Liu EG, Wang DY (2006) Chemical constituents of the bark of Myrica ruba. Central South Pharmacy, 4: 196-199. constituents from bark of Myrica ruba (Lour.) Sieb.et Zucc. Journal of Shenyang Pharmaceutical University 27:34-36.

Lu Q, Wainwright MS, Harris VA, Aggarwal S, Hou Y, Rau T, Poulsen DJ, Black SM (2012) Increased NADPH oxidase-derived superoxide is involved in the neuronal cell death induced by hypoxia-ischemia in neonatal hippocampal slice cultures. Free Radic Biol Med 53(5):1139-51. doi: 10.1016/j.freeradbiomed.2012.06.012. PMID: 22728269.

Mateo I, Infante J, Sánchez-Juan P, García-Gorostiaga I, Rodríguez-Rodríguez E, Vázquez-Higuera JL, Berciano J, Combarros O (2010) Serum heme oxygenase-1 levels are increased in Parkinson's disease but not in Alzheimer's disease. Acta Neurol Scand 121(2):136-8. doi: 10.1111/j.1600-0404.2009.01261.x. PMID: 19785642.

Meotti FC, Luiz AP, Pizzolatti MG, Kassuya CA, Calixto JB, Santos AR (2006) Analysis of the antinociceptive effect of the flavonoid myricitrin: evidence for a role of the Larginine-nitric oxide and protein kinase C pathways. J Pharmacol Exp Ther 316(2):78996. doi: 10.1124/jpet.105.092825. PMID: 16260583.

Park EJ, Jang HJ, Tsoyi K, Kim YM, Park SW, Kim HJ, Lee JH, Chang KC (2013) The heme oxygenase-1 inducer THI-56 negatively regulates iNOS expression and HMGB1 release in LPS-activated RAW 264.7 cells and CLP-induced septic mice. PLoS One 8(10):e76293. doi: 10.1371/journal.pone.0076293. PMID: 24098466. 

22659086.

Piras S, Furfaro AL, Brondolo L, Passalacqua M, Marinari UM, Pronzato MA, Nitti M to oxidative stress in SH-SY5Y neuroblastoma cells. Sci Rep 7(1):7568. doi: 10.1038/s41598-017-08095-7. PMID: 28790431.

Qin X, Cheng J, Zhong Y, Mahgoub OK, Akter F, Fan Y, Aldughaim M, Xie Q, Qin L, Gu L, Jian Z, Xiong X, Liu R (2019) Mechanism and Treatment Related to Oxidative Stress in Neonatal Hypoxic-Ischemic Encephalopathy. Front Mol Neurosci 12:88. doi: 10.3389/fnmol.2019.00088. PMID: 31031592.

Rodrigues Siqueira I, Fochesatto C, da Silva Torres IL, Dalmaz C, Alexandre Netto C (2005) Aging affects oxidative state in hippocampus, hypothalamus and adrenal glands of Wistar rats. Life Sci 78(3):271-8. doi: 10.1016/j.lfs.2005.04.044. PMID: 16112138.

Ten VS, Starkov A (2012) Hypoxic-ischemic injury in the developing brain: the role of reactive oxygen species originating in mitochondria. Neurol Res Int 2012:542976. doi: 10.1155/2012/542976. PMID: 22548167.

Vannucci SJ, Hagberg H (2004) Hypoxia-ischemia in the immature brain. J Exp Biol 207 :3149-54. doi: 10.1242/jeb.01064. PMID: 15299036

Varghese B, Xavier R, Manoj VC, Aneesh MK, Priya PS, Kumar A, Sreenivasan VK (2016) Magnetic resonance imaging spectrum of perinatal hypoxic-ischemic brain injury. Indian J Radiol Imaging 26(3), 316-327. doi.org/10.4103/0971-3026.190421. PMID: 27857456 

miRNA-210 reverses nicotine-induced brain hypoxic-ischemic injury in neonatal rats. Int J Biol Sci 13(1):76-84. doi: 10.7150/ijbs.17278. PMID: 28123348.

Wang Y, Miao Y, Mir AZ, Cheng L, Wang L, Zhao L, Cui Q, Zhao W, Wang H (2016) Inhibition of beta-amyloid-induced neurotoxicity by pinocembrin through $\mathrm{Nrf} 2 / \mathrm{HO}-1$ pathway in SH-SY5Y cells. J Neurol Sci 368: 223-230. doi:10.1016/j.jns.2016.07.010 Wu JH, Huang CY, Tung YT, Chang ST (2008) Online RP-HPLC-DPPH screening method for detection of radical-scavenging phytochemicals from flowers of Acacia 


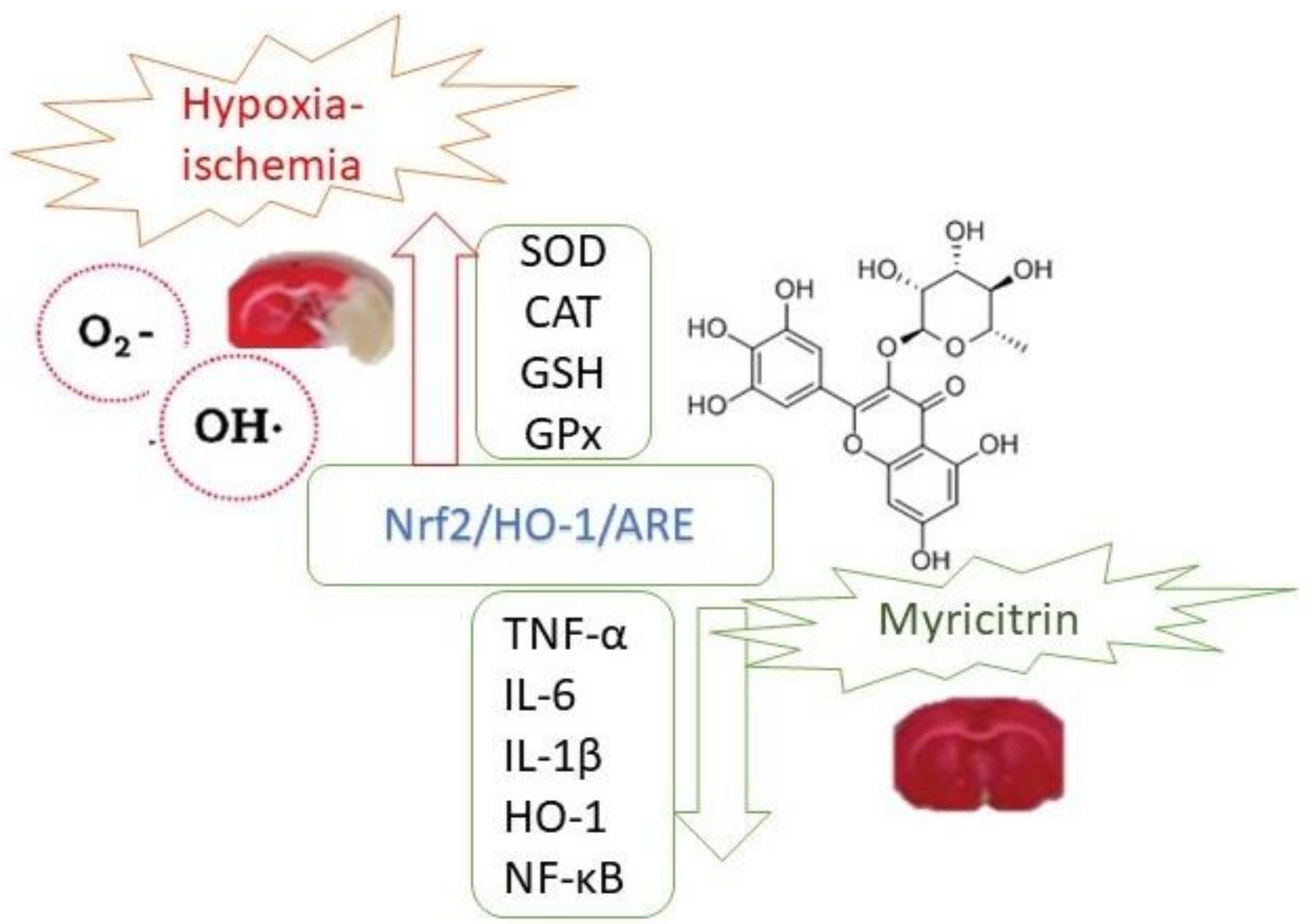




\begin{tabular}{|c|c|c|}
\hline Gene & Primer & Primer \\
\hline Name & Forward (5'-3') & Reverse (5'-3') \\
\hline$\beta$-actin & GGAGATTACTGCCCCTGGCTCCTA & GACTCATCGTACTCCTGCTTGCTG \\
\hline TNF- $\alpha$ & CGGGCTCAGAATTTCCAACA & CGCAATCCAGGCCACTACTT \\
\hline IL-6 & GCCCTTCAGGAACAGCTATGA & TGTCAACAACATCAGTCCCAAGA \\
\hline IL-1及 & CAGGCTTCGAGATGAACAACAA & GTCCATTGAGGTGGAGAGCTTT \\
\hline HO-1 & TGCTCGCATGAACACTCTGGAGAT & ATGGCATAAATTCCCACTGCCACG \\
\hline $\begin{array}{c}\text { NF- } \\
\text { KB/P65 }\end{array}$ & GAGACATCCTTCCGCAAACT & TCCTTCCTGCCCATAATCA \\
\hline
\end{tabular}




\begin{tabular}{|c|c|c|c|c|}
\hline Group & TNF- $\alpha$ (pg/mg) & IL-1ß (pg/mg) & IL-6 (pg/mg) & HO-1 (pg/mg) \\
\hline Sham & $60.31 \pm 3.22$ & $47.69 \pm 2.11$ & $91.40 \pm 1.20$ & $48.16 \pm 1.32$ \\
\hline HI & $128.42 \pm 4.32 * * *$ & $144.54 \pm 8.20 * * *$ & $162.74 \pm 3.13 * * *$ & $112.41 \pm 2.21 * * *$ \\
\hline HI+MY20 & $96.13 \pm 6.28^{\# \# \#}$ & $117.49 \pm 3.97$ \#\#\# & $141.43 \pm 4.64 \#$ & $81.13 \pm 5.54$ \#\# \\
\hline HI+MY40 & $82.69 \pm 7.54$ \#\# & $93.22 \pm 6.84$ \#\# & $128.25 \pm 7.43^{\# \# \#}$ & $68.23 \pm 6.13^{\# \# \#}$ \\
\hline
\end{tabular}

546

547 Table 2. Significant effect of myricitrin on proinflammatory cytokines and HO-1 in of HI 548 induced neonatal rats. Results were expressed as Mean \pm S.D and statistically significant at $\mathrm{P}<0.05$. \#\#\# MY (Myricitrin) treated group compared to HI (Hypoxic ischemic induced group); ***HI group compared with Sham control.

551

552

553

554

555

556

557 
<smiles>C[C@H]1O[C@@H](Oc2c(-c3cc(O)c(O)c(O)c3)oc3cc(O)cc(O)c3c2=O)[C@H](O)[C@@H](O)[C@@H]1O</smiles>

563

564 Figure 1. Molecular structure of myricitrin and molecular formula is C21H20O12 and

565 molecular weight $=464.3763$

566

567

568

569

570

571

572

573 

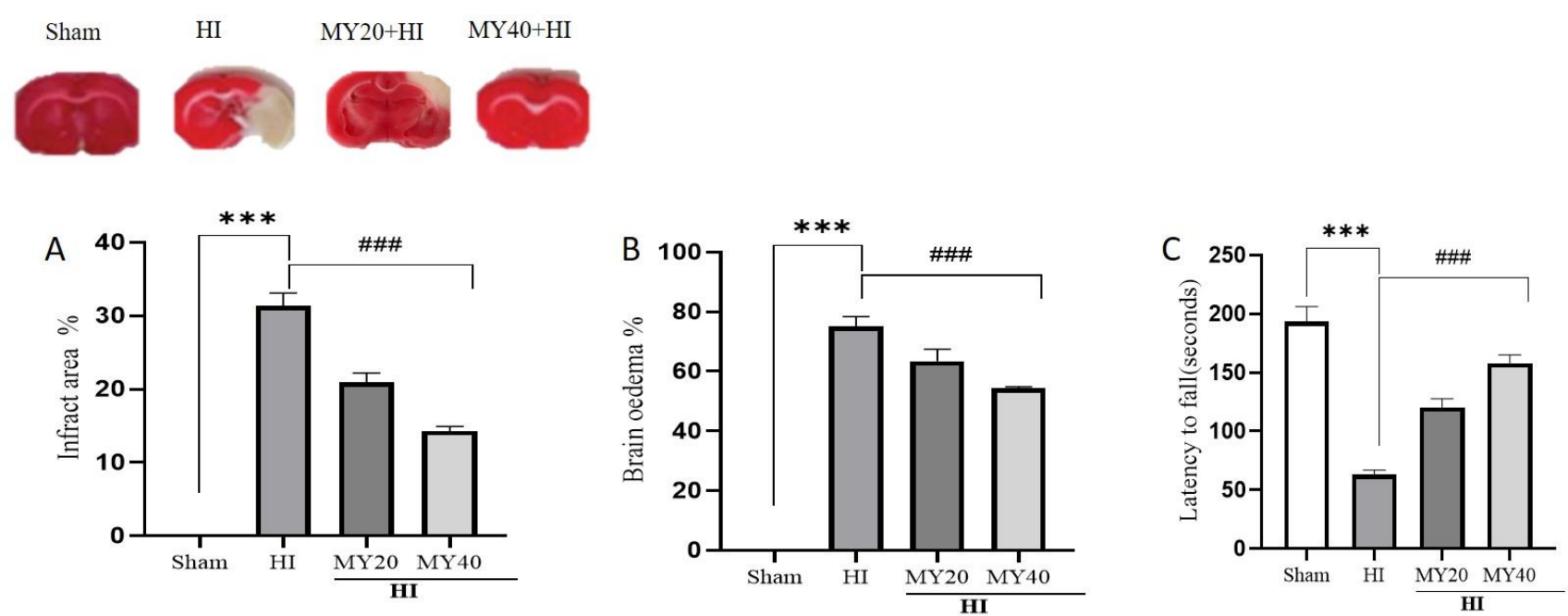

578

579

580

Figure 2. Myricitrin administration diminish infarct volume, oedema (wet weight of brain), improve neurological function studies in hypoxic-ischemic induced neonatal brain injury in a dose-dependent manner.

A, Coronal brain slices stained with TCC shows Infarct area is smaller in myricitrin treated group than $\mathrm{HI}$ group and $\mathrm{n}=6$.

B, Effect of myricitrin on cerebral oedema compared with HI induced neonatal brain and $n=6$

C, RotaRod test was conducted and the expectancy to fall was expressed in seconds shown in with sham, $\# \mathrm{p}<0.05$ compared with MY20 and 40) 

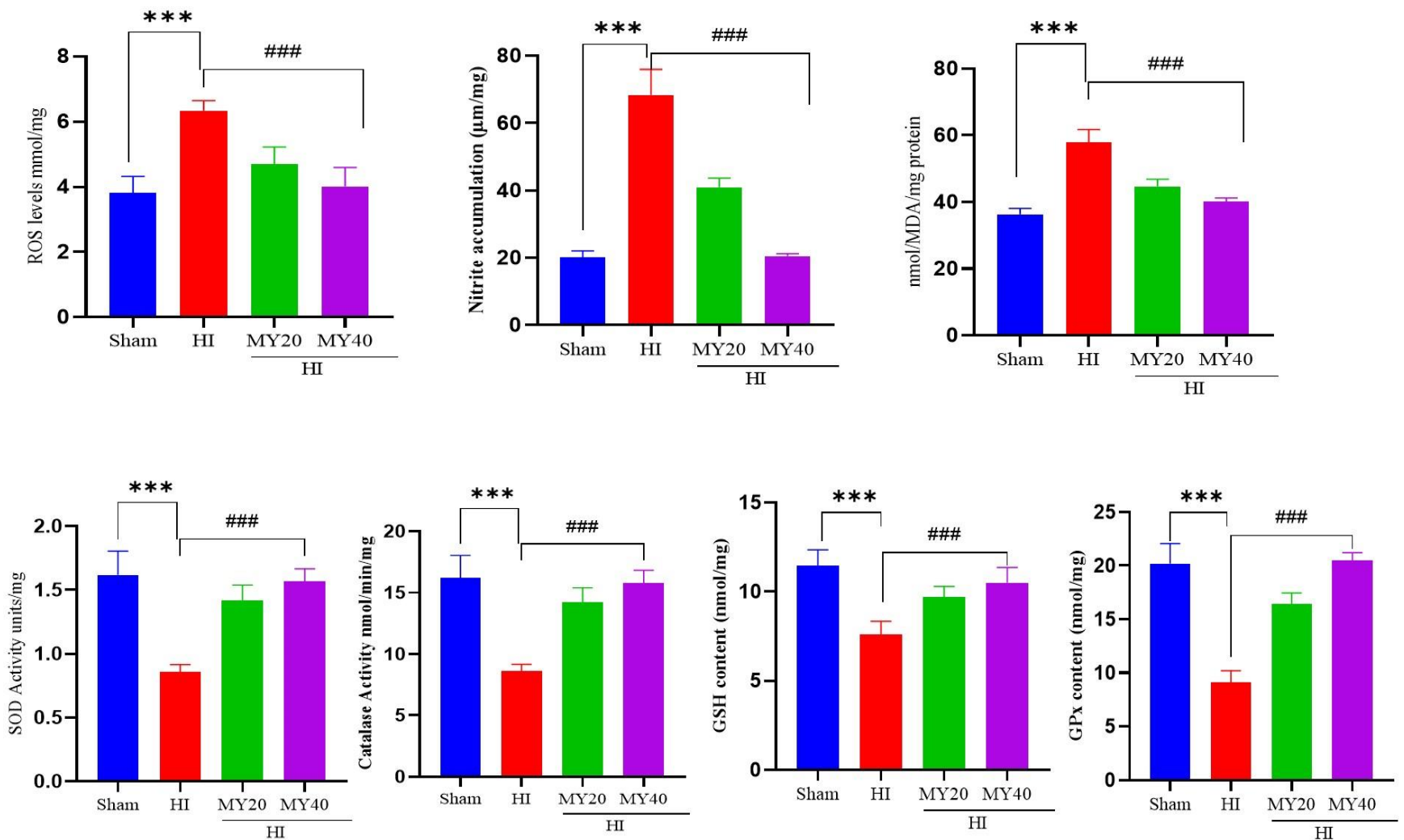

Figure 3. Shows the levels of total ROS, MDA (lipid peroxidation), antioxidant enzymes

598 (SOD, CAT, GSH and GPX) in brain tissues of hypoxic ischemic hemispheres in brain

599 tissues of hypoxic ischemic hemispheres. Results were expressed as Mean \pm SEM and statistically significant at $\mathrm{P}<0.05$. \#\#\# MY (Myricitrin) treated group compared to $\mathrm{HI}$

601 (Hypoxic ischemic induced group); ***HI group compared with Sham control. 

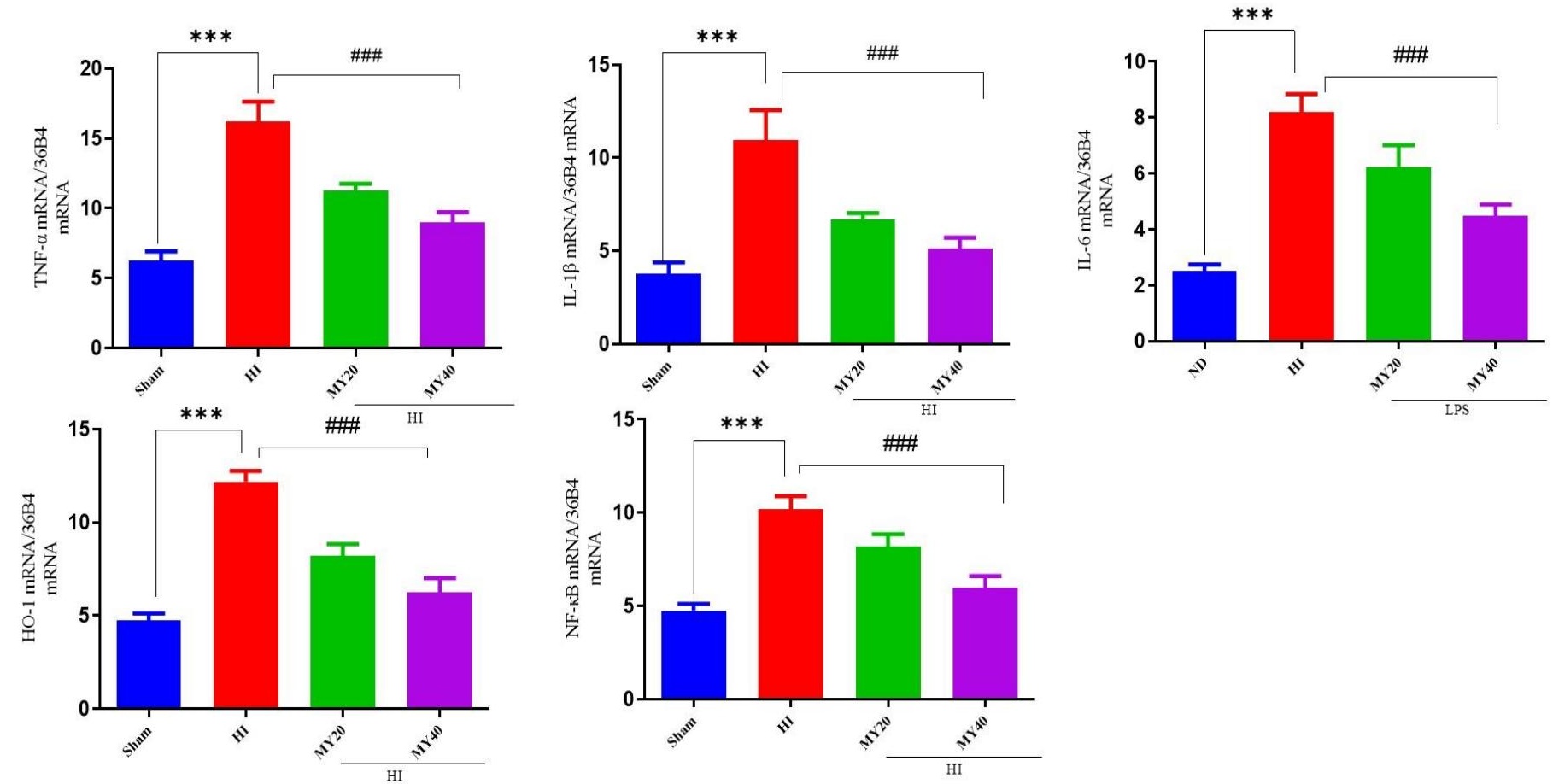

Figure 4. mRNA expression patttern of inflamatory mediators and the results were expressed control. 


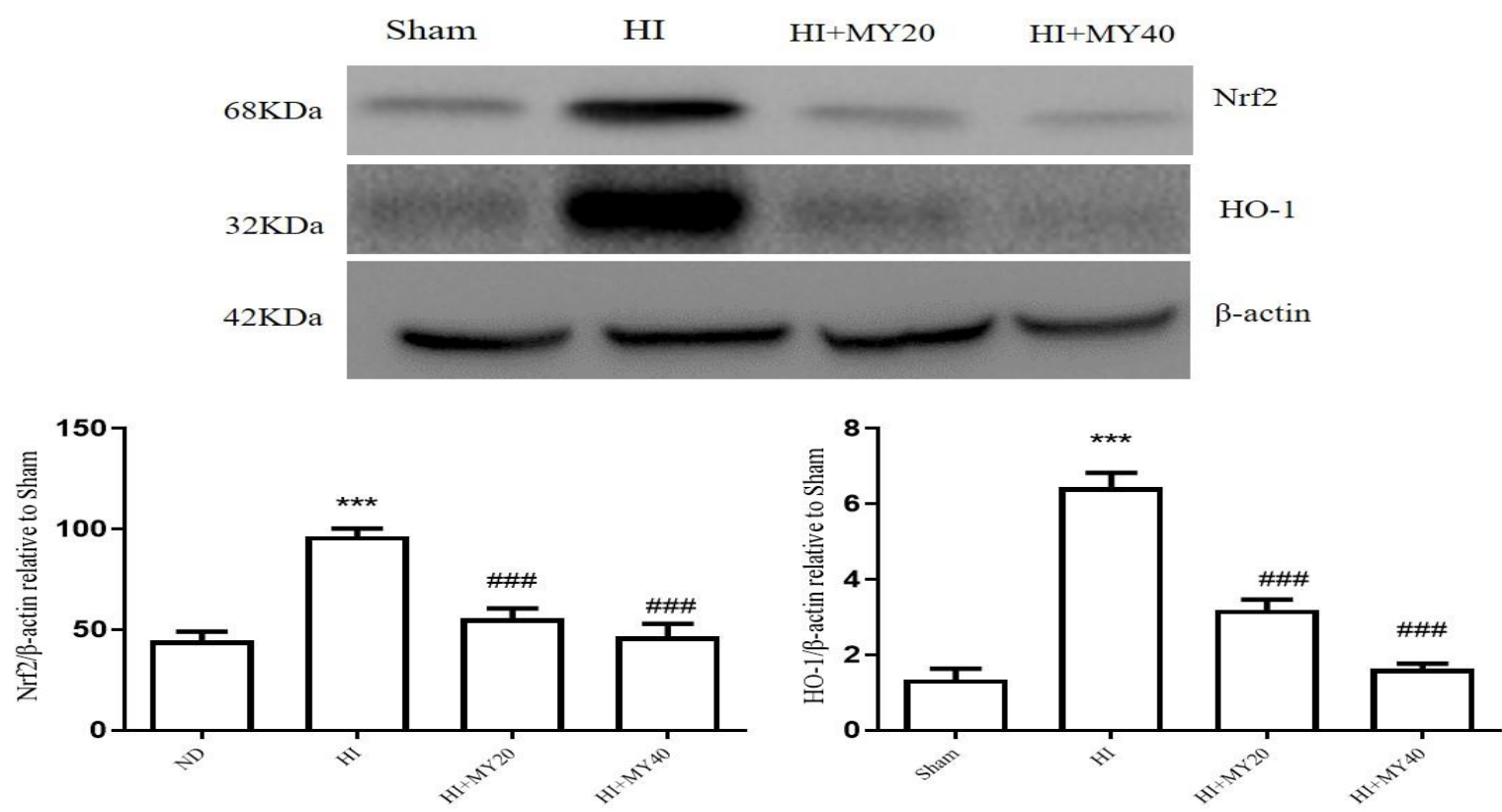

624

625

Figure 5. Figure 5. Myricitrin mediate the over expression of nuclear Nrf2 (A) and cytosolic

626

HO-1 (B) protein in HI induced cerebral cortex of experimental neonatal rats. Results were

627 expressed as Mean \pm SEM and statistically significant at $\mathrm{P}<0.05 . *$ Induced group compared to control; \#\#\# MY (Myricitrin) treated group compared to HI (Hypoxic ischemic induced group); ***HI group compared with Sham control.

630

631 
A

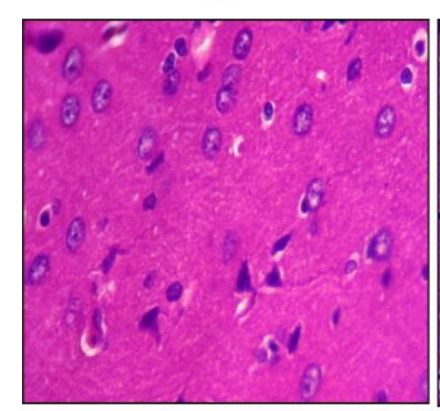

B

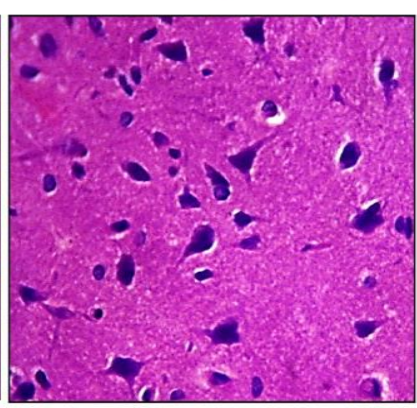

C

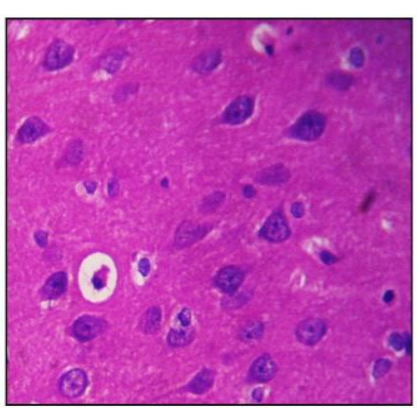

D

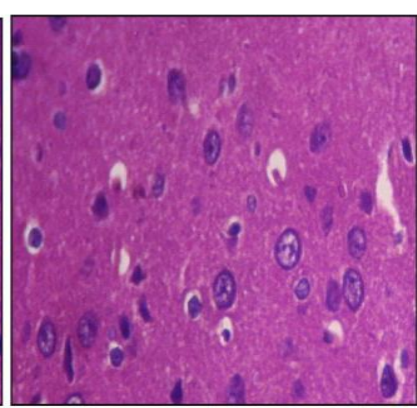

637

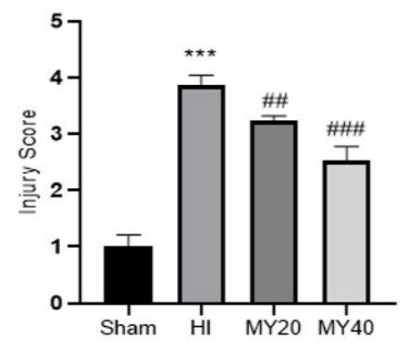

638 Figure 6. Neuroprotective effect of myricitrin observed in HE staining of neonatal cerebral

639 cortex of brain tissue. A, Sham; B, HI; C, MY20+HI; D, MY40+HI. The bar graph shows

640 cell damage scores. Data are expressed as Mean $\pm \mathrm{SD}(\mathrm{n}=6) .{ }^{\# \# \#} \mathrm{P}<0.05$ versus HI group.

641 *** $\mathrm{P}<0.05$ versus Sham group. 
Figures<smiles>C[C@H]1O[C@@H](Oc2c(-c3cc(O)c(O)c(O)c3)oc3cc(O)cc(O)c3c2=O)[C@H](O)[C@H](O)[C@@H]1O</smiles>

Figure 1

Molecular structure of myricitrin and molecular formula is $\mathrm{C} 21 \mathrm{H} 20012$ and molecular weight $=464.3763$
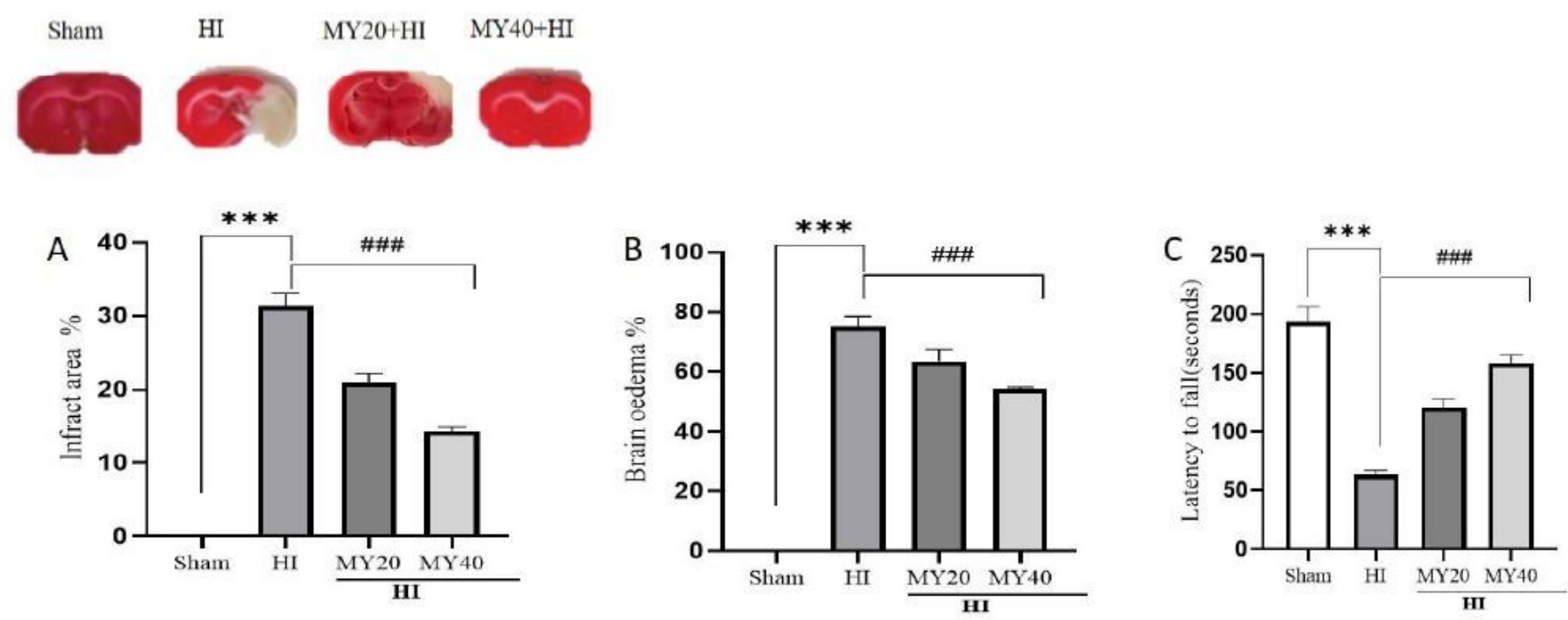

Figure 2 
Myricitrin administration diminish infarct volume, oedema (wet weight of brain), improve neurological function studies in hypoxic-ischemic induced neonatal brain injury in a dose-dependent manner. $A$, Coronal brain slices stained with TCC shows Infarct area is smaller in myricitrin treated group than HI group and $n=6$. B, Effect of myricitrin on cerebral oedema compared with $\mathrm{HI}$ induced neonatal brain and $\mathrm{n}=6 \mathrm{C}$, RotaRod test was conducted and the expectancy to fall was expressed in seconds shown in $\mathrm{C}$ and $n=10$. Statistical analysis data were expressed as the Mean $\pm S D .\left({ }^{*} p<0.05\right.$ compared with sham, $\# p<$ 0.05 compared with MY20 and 40)
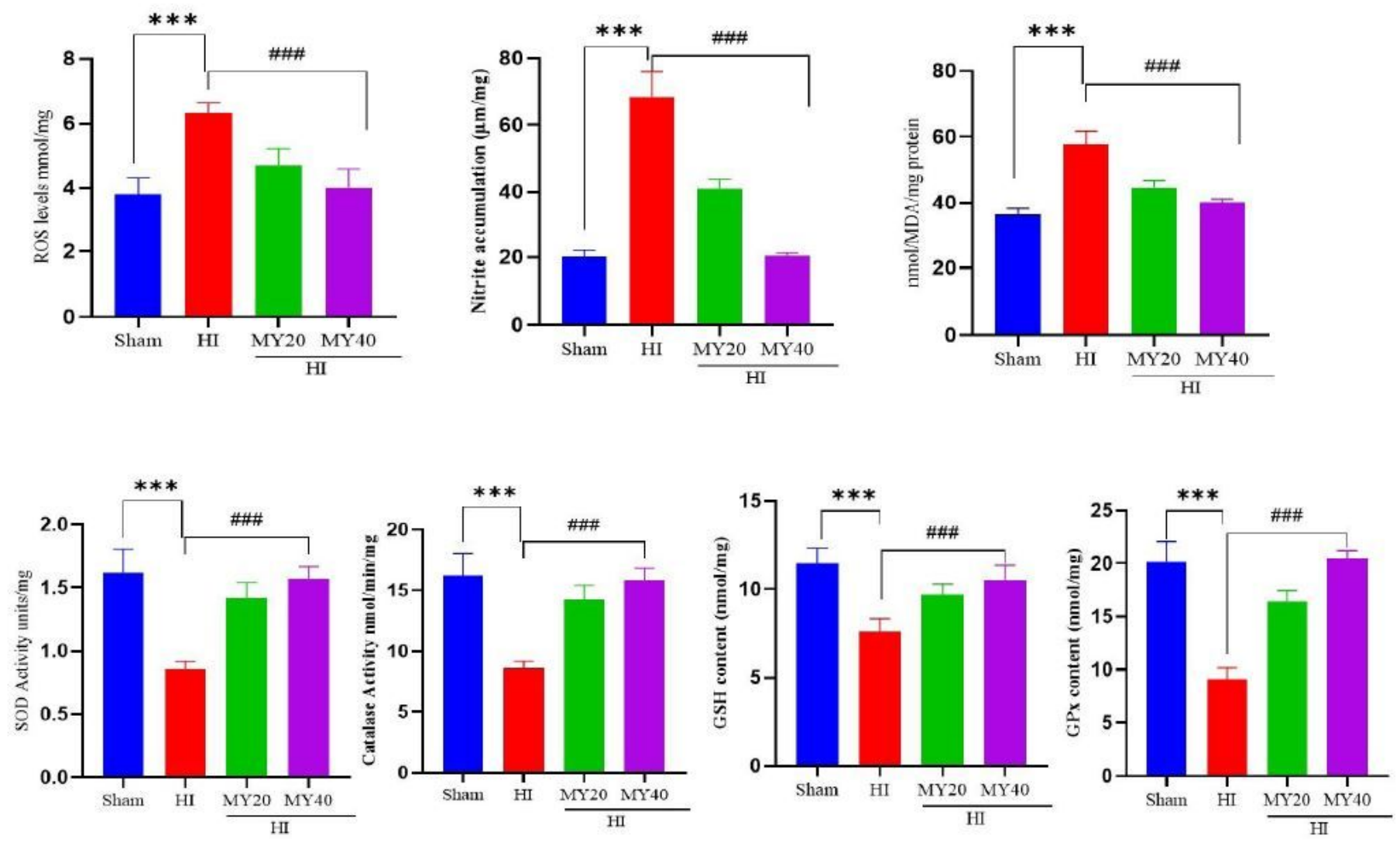

Figure 3

Shows the levels of total ROS, MDA (lipid peroxidation), antioxidant enzymes (SOD, CAT, GSH and GPX) in brain tissues of hypoxic ischemic hemispheres in brain tissues of hypoxic ischemic hemispheres. Results were expressed as Mean \pm SEM and statistically significant at $P<0.05$. \#\#\# MY (Myricitrin) treated group compared to HI (Hypoxic ischemic induced group); ${ }^{* \star *} \mathrm{HI}$ group compared with Sham control. 

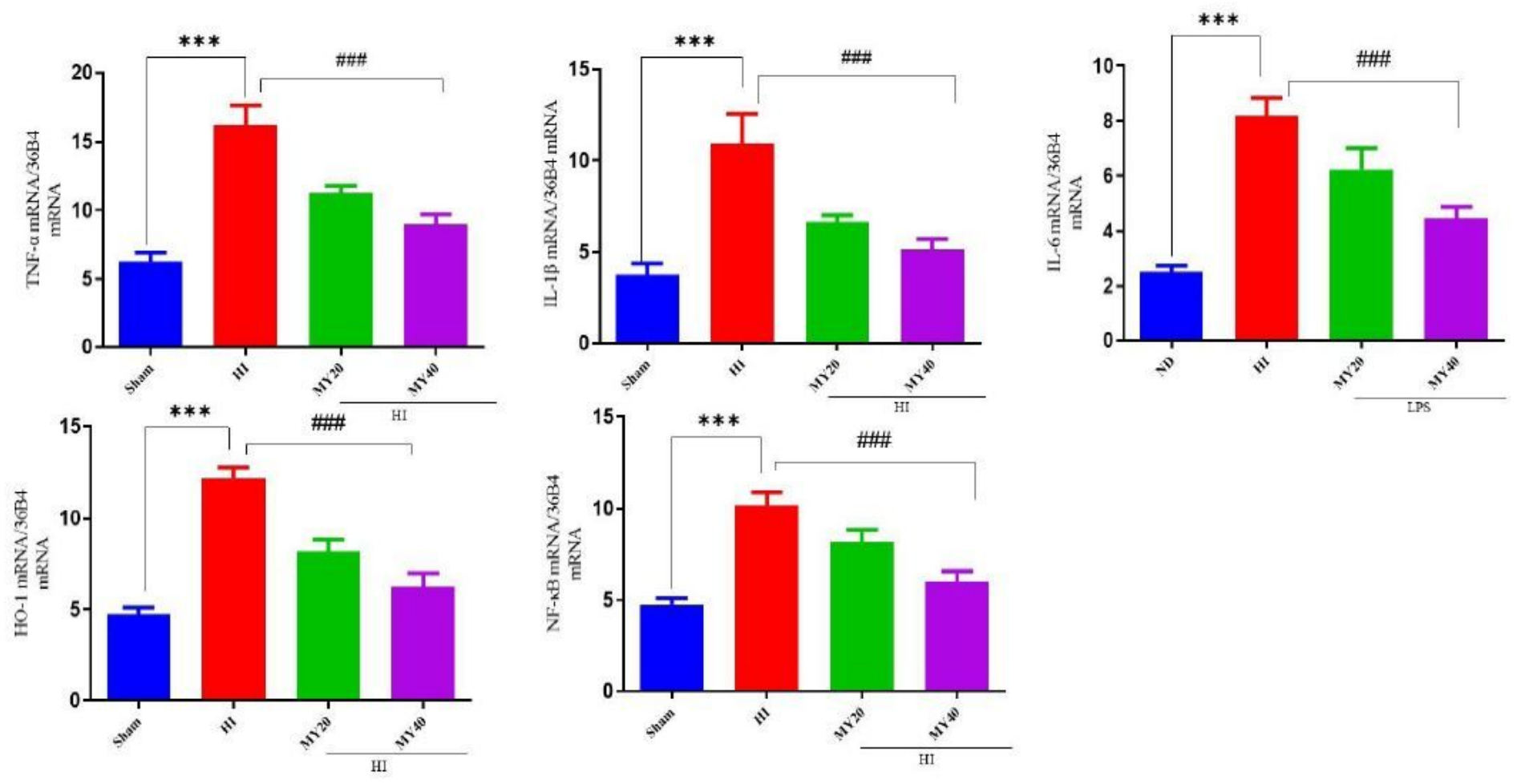

Figure 4

mRNA expression patttern of inflamatory mediators and the results were expressed as Mean \pm SEM and statistically significant at $\mathrm{P}<0.05$. \#\#\# MY (Myricitrin) treated group compared to HI (Hypoxic ischemic induced group); ${ }^{\star \star \star H I ~ g r o u p ~ c o m p a r e d ~ w i t h ~ S h a m ~ c o n t r o l . ~}$ 

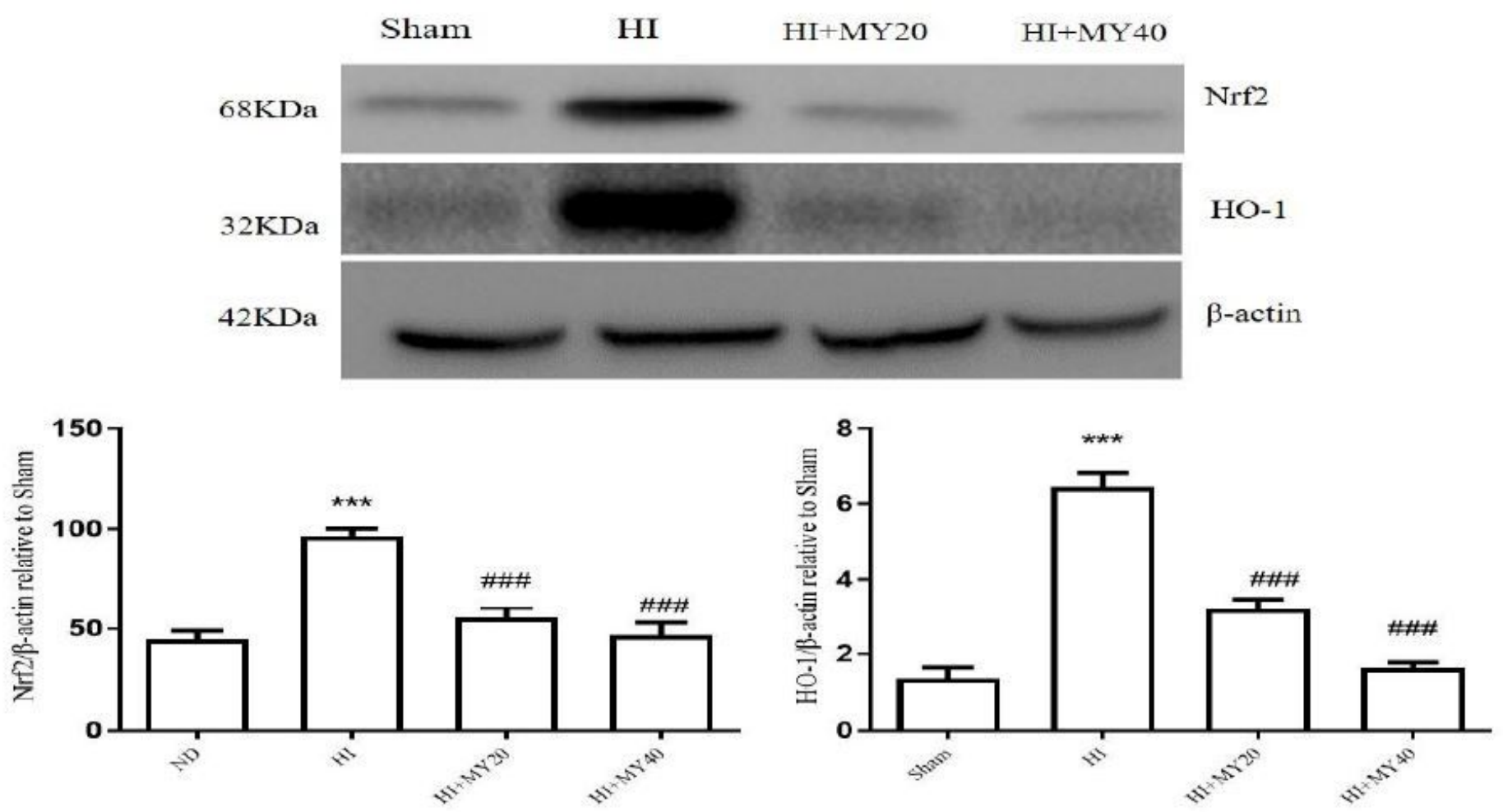

Figure 5

Myricitrin mediate the over expression of nuclear Nrf2 (A) and cytosolic HO-1 (B) protein in HI induced cerebral cortex of experimental neonatal rats. Results were expressed as Mean \pm SEM and statistically significant at $\mathrm{P}<0.05$. * Induced group compared to control; \#\#\# MY (Myricitrin) treated group compared to $\mathrm{HI}$ (Hypoxic ischemic induced group); ${ }^{\star \star \star H H ~ g r o u p ~ c o m p a r e d ~ w i t h ~ S h a m ~ c o n t r o l . ~}$

A
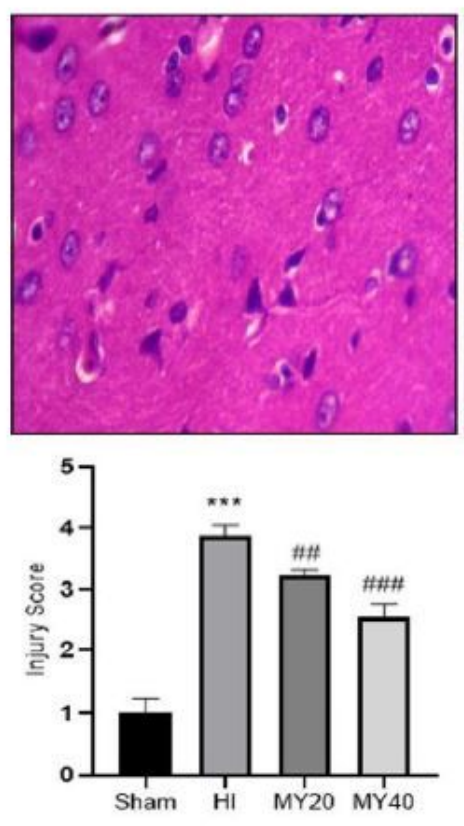

B

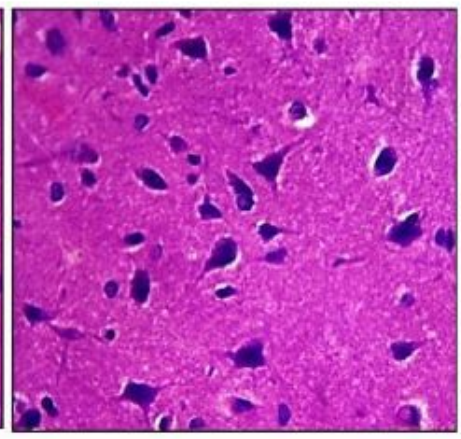

C

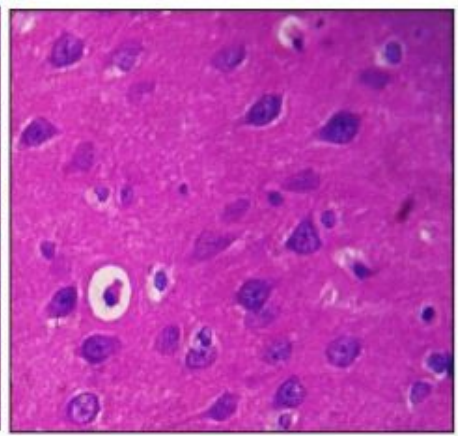

D

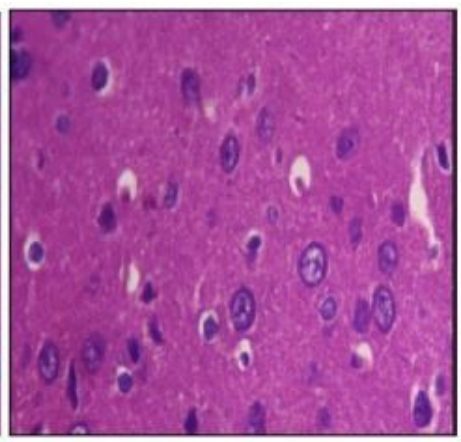


Figure 6

Neuroprotective effect of myricitrin observed in HE staining of neonatal cerebral cortex of brain tissue. A, Sham; B, Hl; C, MY20+Hl; D, MY40+HI. The bar graph shows cell damage scores. Data are expressed as Mean $\pm S D(n=6)$. \#\#\# $P<0.05$ versus HI group. ${ }^{\star \star \star} P<0.05$ versus Sham group.

\section{Supplementary Files}

This is a list of supplementary files associated with this preprint. Click to download.

- Graphicalabstract.JPG

- Graphicalabstract.JPG 